\title{
REPETITIVE TRANSCRANIAL MAGNETIC STIMULATION TREATMENT IN PATIENTS WITH ANOREXIA NERVOSA COMORBID WITH DEPRESSION AND PERSONALITY DISORDER
}

\author{
M. Separovic Lisak ${ }^{1}$, M. Skocic Hanzek${ }^{1}$, Z. Stimac ${ }^{1}$, M. Bajs Janovic ${ }^{1}$, S. Greguras ${ }^{1}$, A. Mihaljevic-Peles ${ }^{1}$ \\ ${ }^{1}$ University Hospital Centre Zagreb, Department of Psychiatry and Psychological Medicine, Zagreb, Croatia
}

Introduction: Anorexia nervosa (AN) is a high mortality disorder in need for new therapeutic approaches as we are still missing a sufficiently effective treatment. Besides, AN is frequently complicated by comorbid anxiety, depression and structural personality disorders. This case series examined the therapeutic potential of repetitive transcranial magnetic stimulation (rTMS) in five female patients with chronic anorexia nervosa, major depressive disorder and personality disorder.

Background: Anorexia nervosa (AN) is complex mental disorder with multiple comorbidities and complications, characterized by weight loss, extreme fear of eating and gaining weight, low body mass index (BMI), distorted body image and emaciation. To this day, there is no FDA approved pharmacotherapeutic treatment for AN. Conventional treatments in terms of psychotherapy, pharmacotherapy and inpatient treatments are largely ineffective and associated with poor response rates and frequent relapse. Transcranial magnetic stimulation (TMS) is a novel treatment, noninvasive brain stimulation method, proved to be effective in reducing depressive symptoms, with developed protocols for treatment of anorexia nervosa. Furthermore, rTMS treatment in borderline personality disorder patients shows various effects including improved self-control, emotion regulation, mood, anxiety, and executive functions.

Materials and methods: Five female patients, age 20-32, with chronic AN, comorbid with borderline personality disorder and depression according to DSM V criteria, received 20 sessions of repetitive transcranial magnetic stimulation with the frequency $10 \mathrm{~Hz}$ to the left dorsolateral prefrontal cortex, 20 trains/day, 50 pulses/train, intertrain interval $55 \mathrm{~s}$ for 20 weekdays. Conventional ED treatment, including atypical antipsychotics and selective serotonin reuptake inhibitors (SSRIs), demonstrated inssuficient effects. Sociodemographic data, medical history, changes in body mass index, eating disorder (ED) symptoms, mood, anxiety and personality structure were assessed via standardized questionnaires and clinical interview pre-treatment, posttreatment and at 6-month follow-up. Clinical instruments that were used: SCID, HAM-D, HAM-A, EAT-26 and BIS 11. Qualitative feedback regarding the intervention was obtained from participants and carers.

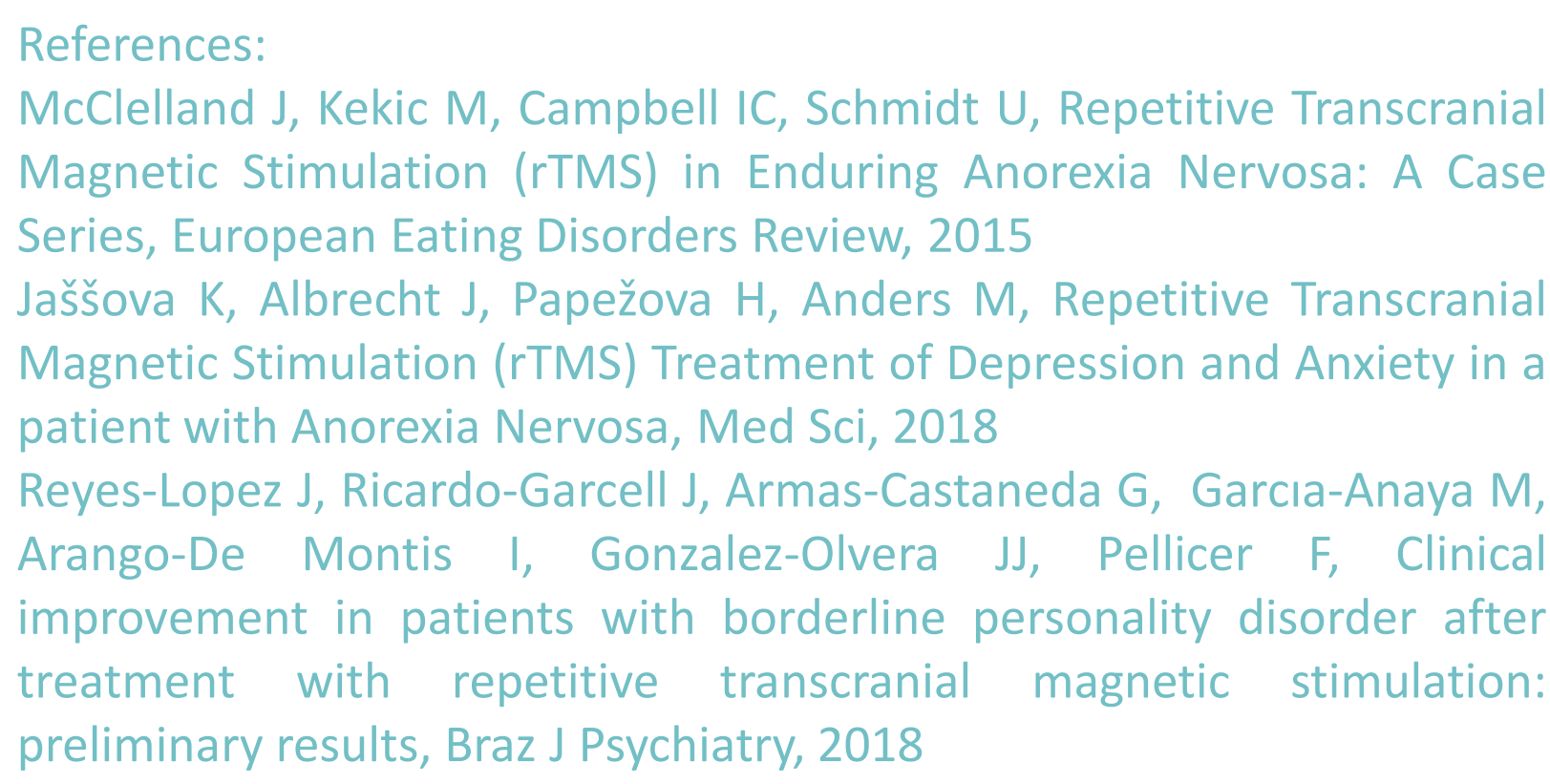

Results: From pre-treatment to post-treatment assessement, affective symptoms and anxiety improved significantly in all participants, and showed further improvements at 6-months follow-up in 4/5. There was a reduction in HAM-A score for $20 \%$ and in HAM-D score for $35 \%$ from the baseline.

At the end of the treatment, ED symptoms and body mass index (BMI) improved in 2/5 participants and remained stable in 3/5. The most prominent change was observed in EAT-26 Oral control subscale. Further improvements in ED symptoms were seen at 6-month follow-up in 3/5 participants.

Two patients showed improvement only on the depression scale but not eating scales, still none of the participants had lost weight.

All the participants showed improved self-control and emotion regulation, more adequate social functioning and better interpersonal relationships.

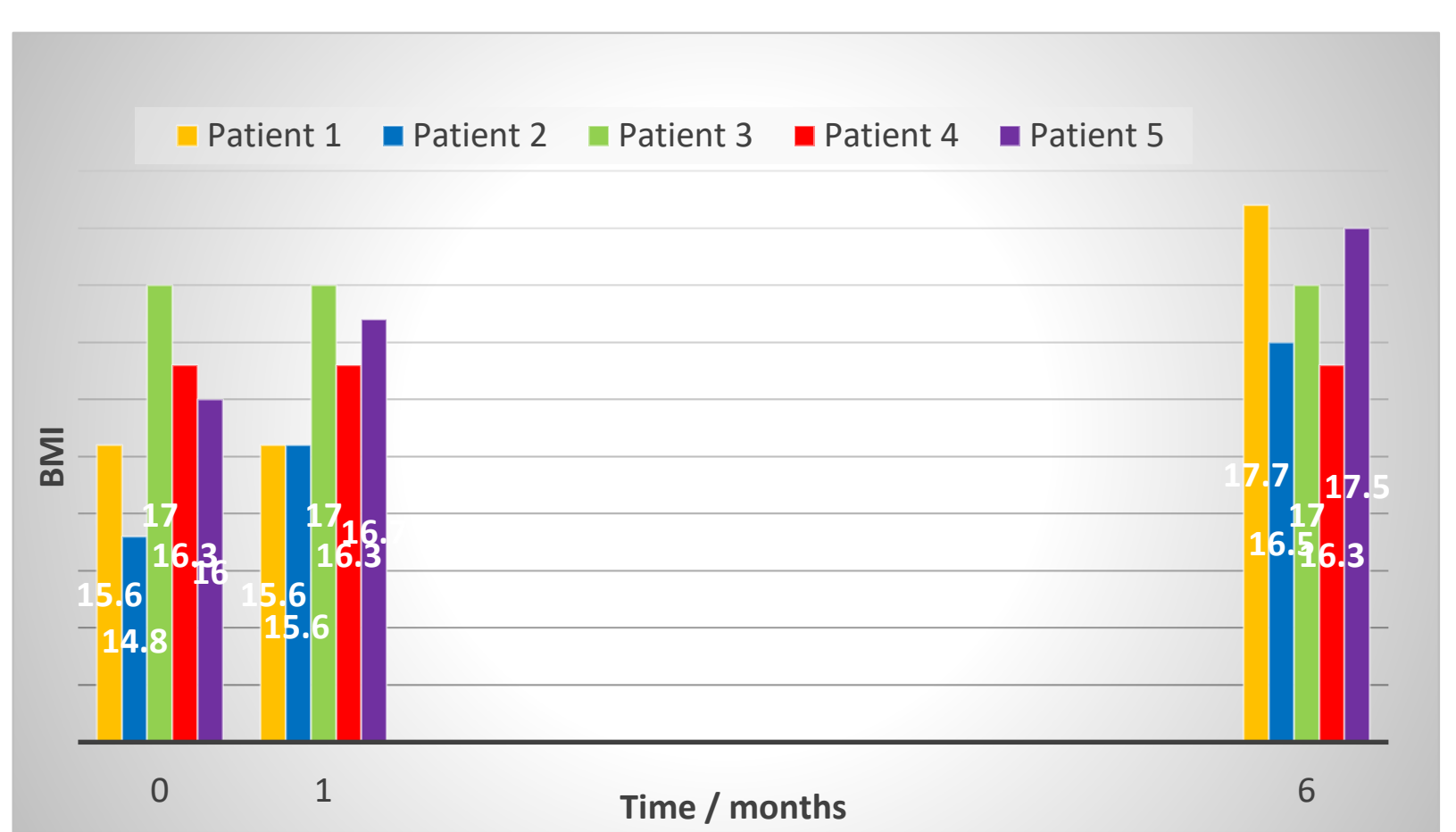

Conclusion: In this case series we showed that, in line with our positive clinical experiences using rTMS therapy in depression, the treatment of AN comorbid with depressive disorder and borderline personality disorder, reduced our patient's anorexic, anxious, and depressive symptoms. The results show that improvements in the mood precede those in ED symptoms which could be due to neuroplastic changes that develop over time. Accordingly, future studies should consider longer follow-up periods. This case study indicates the need for further investigation of therapeutic benefits and efficacy of rTMS in this specific patient population, in particular, targeting optimal brain areas as well as number of rTMS sessions. 